\title{
Bone Quality in Chronic Kidney Disease Patients: Current Concepts and Future Directions - Part II
}

\author{
Kamyar Asadipooya ${ }^{a}$ Mohamed Abdalbary $^{b, c}$ Yahya Ahmad $^{b}$ Elijah Kakani $^{\mathrm{b}}$ \\ Marie-Claude Monier-Faugere ${ }^{b}$ Amr El-Husseini ${ }^{b}$ \\ aDivision of Endocrinology, University of Kentucky, Lexington, KY, USA; ${ }^{b}$ Division of Nephrology \& Bone and Mineral \\ Metabolism, University of Kentucky, Lexington, KY, USA; 'Nephrology and Dialysis Unit, Mansoura University, \\ Mansoura, Egypt
}

\author{
Keywords \\ Chronic kidney disease $\cdot$ Bone quality · Bone mineral \\ density · Management
}

\begin{abstract}
Background: Patients with chronic kidney disease (CKD) have an increased risk of osteoporotic fractures, which is due not only to low bone volume and mass but also poor microarchitecture and tissue quality. The pharmacological and nonpharmacological interventions detailed, herein, are potential approaches to improve bone health in CKD patients. Various medications build up bone mass but also affect bone tissue quality. Antiresorptive therapies strikingly reduce bone turnover; however, they can impair bone mineralization and negatively affect the ability to repair bone microdamage and cause an increase in bone brittleness. On the other hand, some osteoporosis therapies may cause a redistribution of bone structure that may improve bone strength without noticeable effect on BMD. This may explain why some drugs can affect fracture risk disproportionately to changes in BMD. Summary: An accurate detection of the underlying bone abnormalities in CKD patients, including bone quantity and quality abnormalities, helps in institution of ap-
\end{abstract}

propriate management strategies. Here in this part II, we are focusing on advancements in bone therapeutics that are anticipated to improve bone health and decrease mortality in CKD patients. Key Messages: Therapeutic interventions to improve bone health can potentially advance life span. Emphasis should be given to the impact of various therapeutic interventions on bone quality.

(c) 2021 The Author(s) Published by S. Karger AG, Basel

\section{Introduction}

There is an increased risk of fracture in the patients with chronic kidney disease (CKD) [1]. Studies have demonstrated an increased risk of hip fracture over the past decades despite an intensive focus on treatments for renal osteodystrophy (ROD) [2,3]. The bone quantity, quality, and remodeling abnormalities are important factors that determine the treatment method. Treatments only focused on the abnormal bone quantity of CKD are

Part I: Asadipooya et al.: "Bone Quality in CKD Patients: Current Concepts and Future Directions - Part I." Kidney Dis. DOI: $10.1159 / 000515534$. karger@karger.com www.karger.com/kdd

Karger $\stackrel{\text { ' }}{=}$

BOPEN ACCESS
(C) 2021 The Author(s)

Published by S. Karger AG, Basel

This is an Open Access article licensed under the Creative Common Attribution-NonCommercial-4.0 International License (CC BY-NC) (http://www.karger.com/Services/OpenAccessLicense), applicable to the online version of the article only. Usage and distribution for commercial purposes requires written permission.
Correspondence to:

Amr El-Husseini, amr.elhusseini.moh@uky.edu 
thus not likely to be efficacious in preventing fractures due to many other risk factors that affect bone quality and remodeling [4]. There are different nonpharmacological and pharmacological means to improve bone quantity, quality, and function, which lead to better outcomes with a reasonable approach. Therefore, there is a lot to learn about the pathogenesis and treatment of bone disease in CKD patients.

\section{Nonpharmacological Approach}

Bone quality in CKD patients is affected not only by vitamin $\mathrm{D}$ deficiency and hyperparathyroidism but also by comorbidities, such as myopathy, neuropathy, and malnutrition along with inactivity. All these play a role in bone loss, muscle weakness, falls, and fracture. Nonpharmacological approaches, such as smoking cessation, reducing alcohol consumption, weight-bearing/resistance exercise, and physical therapy can technically improve bone quality and muscle power, resulting in reduced falls and fragility fractures [5-7].

Smoking negatively affects bone health by inducing more bone resorption and mineralization defect [8]. In addition, predialysis CKD patients, who are smokers have higher phosphate levels independent of FGF23 and renal function [9]. Concerning the acute detrimental effects of excess alcohol consumption, Asadipooya and Graves reported that excess alcohol consumption caused transient osteoporosis. Alcohol can directly affect bone by reducing osteoblasts and increasing osteoclasts [10]. Moreover, it can indirectly cause systemic alterations, including liver damage, pancreatic damage, muscle atrophy, neuropathy, hormonal changes (PTH, sex hormones, and growth hormone), oxidative stress, and inflammation.

The progression of CKD is associated with physical deterioration, and thus encouraging exercise and rehabilitation is pivotal [11]. However, the optimal level of exercise for CKD patients has not been completely determined. The key points for CKD patients are the individualization of an exercise training program based on baseline functional status, consistency of participation, and assessment of progression [6]. The main concern is cardiovascular status, and it is important to consider the American Heart Association recommendations [12]. It is recommended to start regular exercise slowly 2-3 times a week, as tolerated, and if possible under supervision then increase to 3-5 times a week. This includes weight-bearing, muscle reinforcement, and balance enhancement [6]. Exercise in CKD patients generally improves muscle power, mass, and func- tion. It substantially reduces systemic inflammation, in addition to the improvement of nutrition, body mass index, and BMD in CKD including dialysis patients $[6,13]$. Lean body mass positively correlates with total bone mineral content and BMD in peritoneal dialysis patients [14]. Grzegorzewska and Młot-Michalska [15] reported that total body mass correlated better with femoral neck BMD in dialysis patients than body mass index. However, Fournie et al. [16] revealed that fat mass is negatively correlated with bone quality including cortical and trabecular thickness while lean body mass did not correlate with total volumetric BMD, measured by HR-pQCT. Furthermore, exercise has anabolic effects to prevent muscle wasting [17] and moreover can improve bone formation markers in hemodialysis (HD) patients [18]. Additionally, Marinho et al. [19] reported that BMD significantly improved after 24 weeks of resistance exercise in HD patients.

Exercise, in general, modifies calcium homeostasis and calcium-related hormones, such as PTH, vitamin D metabolites, and calcitonin. It decreases ionized calcium and increases PTH and vitamin D metabolite levels. The change in PTH, which is determined by type, duration, and intensity of exercise, can potentially have bone anabolic effects [20]. It increases bone turnover and metabolism by affecting growth factor signals and endocrine regulators of bone [21]. Furthermore, aerobic exercise during $\mathrm{HD}$ causes peripheral vasodilation and thus hypothetically improves solute removal during dialysis. However, the effects of exercise on dialysis adequacy $(\mathrm{Kt} / \mathrm{V})$ are controversial [22, 23]. Orcy et al. [24] reported that exercise improved phosphate removal without affecting urea, creatinine, and potassium clearance.

Exercise is a downregulator of sclerostin, which inhibits bone formation. Sclerostin levels predicted bone loss in dialysis patients [25] and correlated inversely with physical activity in CKD stage 3 and 4 patients [26]. Nevertheless, exercise in CKD rats is accompanied by a reduction of serum sclerostin and improvement of bone microarchitecture [27]. However, the sclerostin levels did not significantly change after exercise in CKD stage 3-5 patients including those on dialysis $[18,26]$.

High-calorie diet in CKD patients, despite causing weight gain, is associated with less urea generation. In addition, weight loss and malnutrition correlated with worsened outcomes in CKD patients [28]. However, high-fat diets can reduce calcium absorption and consequently elevate $1,25(\mathrm{OH})_{2} \mathrm{D}, \mathrm{PTH}$, and phosphate absorption in CKD patients [28] and experimental animals [29]. An energy-dense diet rich in phosphate can induce a positive phosphate balance and consequently increases FGF23 and 
Table 1. Treatment modalities of mineral bone disorders in CKD patients

Treatment modalities Role in bone metabolism

\section{Nonpharmacological}

Smoking cessation

Limiting alcohol

Exercise as tolerated
Smoking induces bone resorption and mineralization defect Smoking increases phosphorus levels in predialysis CKD patients

Reduces osteoblast and increases osteoclast Systemic alterations

Anabolic effects: prevents muscle wasting, improves BMI and BMD

Reduces inflammation

Improves $\mathrm{Ca}$ and $\mathrm{PO} 4$ homeostasis

Experimentally improves bone microarchitecture in CKD rats

Downregulates sclerostin production
Diet (micronutrients, vitamins, antioxidants, plant-based food, fibers, polyunsaturated fatty acids, and Mediterranean)
Pro-inflammatory diet (high-calorie nutrients) is associated with lower BMD and higher fracture risk

Plant sources of proteins can help bone collagen without inducing acid load Low-protein diet with ketoanalogues may help CKD parameters

Pharmacological

Phosphate-lowering therapies (calcium carbonate, calcium acetate, sevelamer, lanthanum, tenapanor)
First line in CKD patients

Improve SHPT and BMD in CKD patients

Calcium containing binders may induce LTBD more than sevelamer and lanthanum

Lanthanum improves bone turnover, bone volume, mineralization of periosteal surface and endocortical surface in dialysis patients

Vitamin D and VDRA (calcitriol, paricalcitol, doxercalciferol, alfacalcidol)

First-line therapy in CKD with SHPT and vitamin D deficiency

Higher risk of hypercalcemia and hyperphosphatemia with VDRAs than vitamin D

Maintaining vitamin $\mathrm{D}$ at a balanced level, even combination of vitamin $\mathrm{D}$ and VDRA is helpful for SHPT and bone markers

VDRAs may induce LTBD

Control SHPT and fracture risk

Decrease high BTMs toward normal

Etelcalcetide might be more effective in reducing the bone turnover in patients with severe SHPT

Might have PTH independent anabolic bone effects

\section{Antiresorptive therapies}

BPs

Denosumab

Gonadal hormones and SERM (sex hormones, raloxifene, and bazedoxifene)

Mainly studied in osteoporotic early CKD patients without evidence of LTBD Longer half-life in advanced stages of CKD and may induce LTBD Decrease bone loss with less impact on bone quality

Not renally excreted and so does not accumulate in CDK patients

Safely improves BMD and reduces fractures in postmenopausal women with CKD stage $1-4$

Increases BMD and decreases iPTH in dialysis patients with iPTH $>1,000 \mathrm{pg} / \mathrm{mL}$ Reduces bone turnover more than BPs Might cause profound hypocalcemia especially in advanced CKD

Raloxifene increases BMD and improves bone quality in postmenopausal women with CKD

Bazedoxifene improves renal function, $\mathrm{BMD}$, and phosphate excretion in postmenopausal women

Bazedoxifene reduces BTMs and fractures in postmenopausal women with CKD Similar vertebral fractures risk reduction compared to BPs

Transdermal HRT in premenopausal dialysis women improves lumbar spine BMD

Calcitonin

Combined with vitamin $\mathrm{D}$, increases BMD

Calcitonin prevents the bone loss after kidney transplant 
Table 1 (continued)

\begin{tabular}{ll}
\hline Treatment modalities & Role in bone metabolism \\
\hline Anabolic therapies & $\begin{array}{l}\text { Low doses can stimulate bone formation, but high doses may cause } \\
\text { mineralization defect/osteomalacia in CKD patients }\end{array}$ \\
\hline Teriparatide and abaloparatide & $\begin{array}{l}\text { Improve bone formation in patients with LTBD } \\
\text { Improve or maintain lumbar spine BMD } \\
\text { Reduce the fracture rate in postmenopausal women with osteoporosis and mild } \\
\text { to moderate CKD }\end{array}$ \\
\hline Romosozumab & $\begin{array}{l}\text { Not studied in CKD patients, and there is a concern of increased extraskeletal } \\
\text { calcification } \\
\text { Alendronate might have a protective impact in reducing romosozumab CV } \\
\text { events }\end{array}$ \\
\hline New therapies & $\begin{array}{l}\text { Provide potential role as antiresorptive therapy in metabolic bone disorders } \\
\text { Was not approved by FDA, due to a concern of increased risk of cerebrovascular } \\
\text { events }\end{array}$ \\
\hline Cathepsin K antagonists & $\begin{array}{l}\text { Approved by the FDA for treatment of X-linked hypophosphatemia } \\
\text { May lead to hyperphosphatemia in early CKD patients } \\
\text { Not enough evidence to use in advanced CKD patients }\end{array}$ \\
\hline Anti-FGF23 antibodies & \\
\hline
\end{tabular}

CKD, chronic kidney disease; LTBD, low turnover bone disease; VDRA, vitamin D receptor activator; P, phosphate; FGF23, fibroblast growth factor 23; VDRA, vitamin D receptor activator; BPs, bisphosphonates; SERMs, selective esterogen receptor modulators; BMI, body mass index.

PTH and decreases calcitriol levels [28]. Diet that includes certain micronutrients, vitamins, antioxidants, plantbased food, fibers, polyunsaturated fatty acids, and Mediterranean diet is associated with better bone health and less osteoarthritis [30]. They even can lower the risk of CKD and delay CKD progression [31]. This can impact bone health through improvement of calcium, phosphate, and PTH regulation. Contrarily, high-calorie nutrients including trans fat, saturated fat, and cholesterol can lead to chronic inflammation. This can potentially worsen the bone health [30]. The effects of high-protein diet with high endogenous acid production and chronic metabolic acidosis on BMD and fracture are controversial [32, 33]. Plant rather than animal sources of proteins can help bone collagen without inducing more acid load. However, a meta-analysis did not show a benefit of soy protein versus animal protein on BMD, BTMs, and fracture [34]. Nevertheless, most studies were observational studies or interventional trials with a small number of enrollees, and hence further studies warranted to clarify the exact effects of diet on bone health. Ascorbic acid supplements transiently increased serum calcium level, without significantly affecting serum phosphate and PTH levels. Its use in CKD patients is not supported [35].
In a recent meta-analysis, low-protein diet supplemented with keto-analogs seemed to help kidney function and CKD-MBD parameters [36]. Furthermore, keto-analog supplementation in CKD patients is associated with improvement of bone metabolism and insulin sensitivity [37]. This might be similar to the effects of low-protein and vegetarian diet in CKD patients [38]. The pharmacological and nonpharmacological bone quality interventions in CKD patients are summarized in Table 1.

\section{Pharmacological Approach}

Hormonal and biomarker changes are the main targets of pharmacological approaches in CKD-MBD patients. In addition, we must consider the state of bone turnover, bone density, potential of mineralization defect, microarchitecture changes, and other indicators of bone quality changes, together with the responsible mechanisms to be able to institute the right therapeutic approach. Herein, we are trying to focus on the impact of various therapeutic interventions on bone quality in CKD patients. The mechanisms of action of bone pharmacotherapeutics are illustrated in Figure 1. 


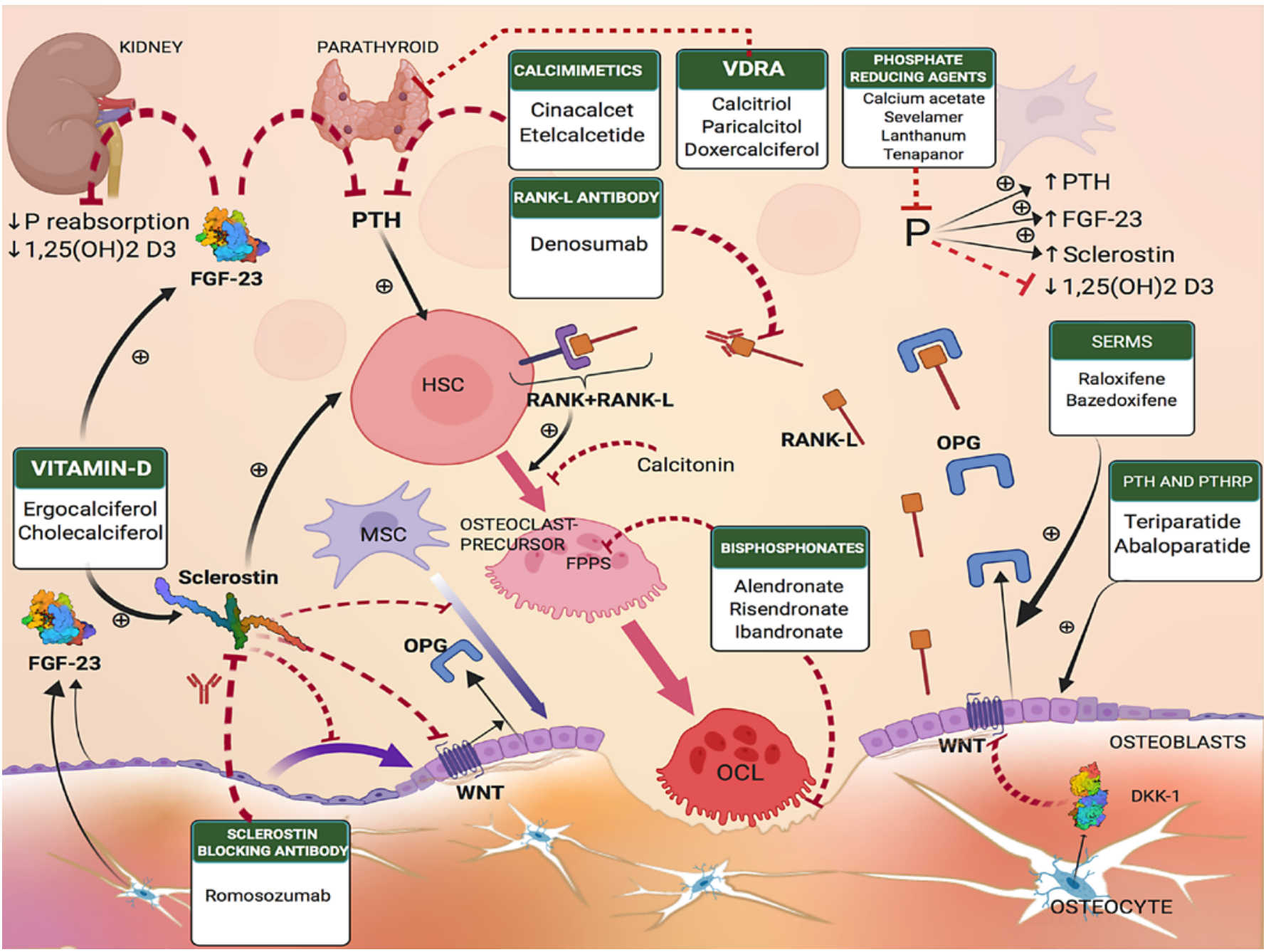

Fig. 1. Mechanisms of action of bone pharmacotherapeutics: vitamin D stimulates the release of 23 FGF23 and sclerostin from osteocytes. Sclerostin has an inhibitory effect on Wnt signaling and OPG production from osteoblasts. Romosozumab blocks sclerostin. DKK1 is another inhibitor of the Wnt pathway. OPG is a decoy receptor that binds RANK-L and prevents stimulation of OCLs. FGF23 inhibits phosphate reabsorption in the kidneys, decreases activation of vitamin D and PTH release. Calcimimetics and VDRA inhibit the production of PTH. P has a stimulatory effect on PTH, FGF23 as well as sclerostin release and an inhibitory effect on activation of vitamin D. Denosumab binds RANK-L and consequently inhibits osteoclastic differentiation. BPs inhibit the maturation of osteoclast precursors and bone resorption. SERMs act on osteoblasts and stimulate the production of OPG and consequently in-

\section{Calcium Supplements}

The use of calcium supplements was advocated because of their possible benefits to bone health. In a double-blinded, placebo-controlled trial, increased habitual calcium intake lowered markers of bone turnover [39]. In hibit osteoclast function. Teriparatide and abaloparatide have anabolic effects on bone and stimulate both osteoblastic activity and bone turnover. Arrow with plus sign denotes stimulatory effect; red dotted line indicates inhibitory effect; arrow without plus sign denotes expression or release of a substance. MSCs; RANK-L receptor (RANK); 1,25 di-hydroxy vitamin D3 (1,25 [OH]2 D3); HSCs; FPPS; and PTHRP. P, phosphate; FGF23, fibroblast growth factor 23; OPG, osteoprotegerin; DKK1, dikkopf-related protein 1; RANK-L, receptor activator of nuclear factor kappa B ligand; OCL, osteoclasts; PTH, parathyroid hormone; VDRA, vitamin D receptor activator; BPs, bisphosphonates; SERMs, selective esterogen receptor modulators; MSCs, mesenchymal stem cells; HSCs, hematopoietic stem cells; FPPS, farnesyl pyrophosphate synthase; PTHRP, parathyroid hormone-related protein analog. 
There was a misconception that CKD patients are necessarily calcium deficient but there is a strong evidence that they are in a positive calcium balance, especially those on high-calcium diet [41] or calcium supplements [42]. Moreover, positive calcium balance can lead to extraskeletal deposition of calcium in the myocardium, small, and large arteries leading to increased cardiovascular risk [43]. Possibility of positive calcium balance and increased extraskeletal calcification should be taken in consideration while prescribing calcium supplements to CKD patients.

\section{Phosphate Binders}

Hyperphosphatemia participates in the development of bone fragility directly by suppressing 1-alpha-hydroxylase activity and indirectly by increasing PTH, FGF23, and sclerostin levels [44]. There is a positive correlation between serum phosphorus levels and fracture risk in healthy subjects [45] and CKD patients [44, 45]. Of note, posttransplant hypophosphatemia has also been shown to predispose to osteoporosis by impairing osteoblastogenesis and inducing osteoblast apoptosis [46].

The KDIGO guideline recommends lowering serum phosphorus levels toward the normal range in CKD patients by dietary phosphate restriction and using phosphate-lowering therapies. Treatment of hyperphosphatemia inhibits the oversecretion of PTH and the development of high turnover bone disease (HTBD). There are different forms of phosphate-lowering therapies, including calcium-containing (calcium carbonate and calcium acetate) and noncalcium-containing binders (sevelamer, lanthanum, ferric citrate, and sucroferric oxyhydroxide). More recently, tenapanor (inhibitor of intestinal sodium/ hydrogen exchanger 3, which blocks paracellular transport of phosphate from the intestinal lumen) has been approved for the treatment of hyperphosphatemia in CKD patients [44].

Phosphate binders have been shown to have variable effects on BMD in moderate CKD patients. Calcium-containing phosphate binders have been shown to decrease [47] or increase BMD [48]. However, Block et al. [48] reported that phosphate binders when evaluated together (calcium acetate, lanthanum carbonate, and sevelamer carbonate) improved annual lumbar spine BMD measured by QCT. It is unclear if these changes in BMD translate to improvement in bone quality and fracture risk.

Raggi et al. [47] reported that calcium-containing phosphate binders in HD patients led to a decrease in thoracic vertebral trabecular and cortical bone attenuation measured by CT. This was associated with lower bone turnover compared to sevelamer in this study. The data about cor- relations between calcium-based phosphate binders and low turnover bone disease (LTBD) are inconsistent [4951]. Barreto et al. [50] and Ferreira et al. [51] found no difference in bone turnover or other bone histopathologic parameters using calcium containing phosphate binders compared to sevelamer in dialysis patients. However, Ferreira et al. [51] observed no differences in mineralization, but increase in bone formation besides improvement of trabecular architecture in sevelamer treated patients.

We have previously reported that lanthanum leads to higher bone turnover and volume in HD patients in comparison to calcium-containing phosphate binders [49]. This can be related to an improvement $[52,53]$ or prevention [54] of LTBD in HD patients. Zhang et al. [55] reported that in diabetic dialysis patients with LTBD, lanthanum improved BMD accompanied by an increase in BTMs and iPTH compared to calcium carbonate. Lanthanum increases mineralization of periosteal surface and endocortical surface [53]. Furthermore, the BMD improvement could be due to accumulation of lanthanum in bone [52], although other studies reported this deposition is negligible $[49,56]$. The long-term effects of lanthanum on fracture risk in CKD patients are unclear.

\section{Vitamin D and Vitamin D Receptor Activators}

Vitamin D attracted considerable attention because of its roles in calcium homeostasis, bone and muscle metabolism, inflammation, and PTH [57]. CKD patients have higher prevalence of vitamin $\mathrm{D}$ deficiency, which is not only associated with SHPT and HTBD but also correlates with low BMD, muscle weakness, and increased risk of falls and fracture [58]. Vitamin D deficiency in CKD patients reduces bone formation, increases subperiosteal resorption, and leads to bone demineralization and bone loss [59]. Vitamin D supplementation is a first-line therapy in CKD patients with low vitamin D levels and SHPT. It has a little effect on PTH and BTMs and is associated with lower risk of hypercalcemia and hyperphosphatemia than vitamin D receptor activators (VDRAs) [58]. In addition, activation of vitamin $\mathrm{D}$ seemed to continue even in dialysis patients, as supplementation with native vitamin $\mathrm{D}$ in $\mathrm{HD}$ patients increased $1,25(\mathrm{OH})_{2} \mathrm{D}$ levels [60]. This could be due to extrarenal activity of 1-alpha-hydroxylase [58]. Of note, observational studies are the main source of the justification of vitamin D supplementation in CKD patients and the optimal level besides its effect on bone density, architecture, quality, and fracture needs more studies. Remarkably, African Americans with CKD had lower 25(OH) $\mathrm{D}$ and higher PTH levels [61], but higher BMD [62] and lower fracture rate compared to Whites [63]. 
VDRAs (calcitriol, alfacalcidol, paricalcitol, and doxercalciferol) can effectively reduce PTH and improve bone histomorphometry in CKD stage 3-5 patients [64]. Six-month use of paricalcitol increased lumbar spine $\mathrm{BMD}$, decreased bone remodeling, and mineral loss along with reducing $\mathrm{PTH}$ in kidney transplant recipients with SHPT [65]. Their effects on PTH reduction was less compared to cinacalcet and they may lead to hypercalcemia, hyperphosphatemia, elevation of FGF23 [64], and increase in sclerostin level [66]. In addition, VDRA can induce LTBD [64]. In a small 12-month placebo-controlled RCT, calcitriol changed the spectrum of the bone disease from HTBD to LTBD in CKD patients prior to dialysis [67]. Furthermore, there is a potential risk of coronary calcification with VDRA use [68].

Generally, vitamin D should be kept at well-balanced levels to maintain the structural integrity of bone, especially in CKD patients [58]. Vondracek and Hoody recommended maintaining 25-OH vitamin $\mathrm{D}$ levels between 30 and $50 \mathrm{ng} / \mathrm{mL}$, with native vitamin D and/or VDRA in advanced CKD patients. They highlighted the need for RCTs evaluating the safety and efficacy of combination therapy [69].

Calcimimetics (Cinacalcet, Evocalcet, and Etelcalcetide)

Calcimimetics reduce PTH synthesis and secretion by enhancing the sensitivity of calcium-sensing receptors in parathyroid glands leading to reduction of high $\mathrm{PTH}$-induced outflow of calcium and phosphate from bone [70]. Cinacalcet has favorable effects on HTBD in dialysis patients. It reduced PTH, BTMs, and improved histological parameters of bone turnover [71]. The effect of cinacalcet on fracture is uncertain, but it decreases the need for parathyroidectomy [72]. Moreover, it has a better PTH reduction efficacy than vitamin $\mathrm{D}$ and VDRAs in CKD or HD patients with SHPT $[73,74]$.

Etelcalcetide is the first FDA approved injectable calcimimetic. Etelcalcetide was superior to placebo and even other calcimimetics (cinacalcet and evocalcet) in the reduction of PTH in CKD patients with SHPT [74], with more pronounced reductions in serum FGF23 and BTMs than placebo [75] and cinacalcet [76]. Moreover, its effect was sustained with no new safety concerns after its use for 1 year [77]. It may improve adherence to therapy with progressively declining $\mathrm{PTH}$, phosphorus, and alkaline phosphatase over 1-year of treatment [78]. Etelcalcitide effectively lowered serum FGF23 and BTM levels in a post hoc analysis of a Japanese multicenter study in HD patients with SHPT [79]. To date, there is no clinical data regarding the impact of etelcalcetide on bone histopathology.

Improving Bone Quality in CKD Patients
However, it is experimentally demonstrated that etelcalcetide enhanced osteoblast activity through a non-PTHdependent pathway [80], besides reducing bone turnover, mineralization defect, and marrow fibrosis with favorable effects on bone structure and strength [81]. It is debatable if calcimimetics exert direct effects on bone. This might open new clinical perspectives to study the impact of various forms of calcimimetics on bone in CKD patients.

\section{Antiresorptive Therapies}

Bisphosphonates

Bisphosphonates (BPs) accumulate in the active bone remodeling sites, usually during enhanced bone turnover, increase osteoclast apoptosis, and thus suppress bone resorption. They are mainly used in osteoporosis management in nonadvanced CKD patients without evidence of LTBD. Since BPs are cleared by the kidney, they accumulate in CKD patients and can induce LTBD. Furthermore, several studies reported that some BPs, particularly zoledronic acid, have deleterious effects on kidney function. They can induce collapsing focal segmental glomerulosclerosis and/or tubular toxicity [82]. This may be reversible upon discontinuation of medication or switching to other antiresorptives, such as denosumab [83]. Moreover, they also increase sclerostin production that might decrease bone formation through inhibition of the Wnt signaling pathway [84]. However, they increase serum PTH level in postmenopausal HD patients [82]. Alarkawi et al. [85] in a large recent retrospective study reported that BPs decreased fracture risk and may have a survival benefit in advanced CKD patients with prior history of fractures.

In kidney transplant recipients, BPs increased lumbar spine and femoral neck BMD [86], but their outcomes on fracture reduction were heterogenous $[86,87]$. In a pooled analysis of 9 trials, risedronate improved BMD, except at the femoral neck in patients with severe CKD. In addition, it reduced vertebral fractures in CKD patients [88]. To the contrary, in a meta-analysis BPs did not lower the fracture rate among kidney transplant recipients and stage 3 or 4 CKD patients [87]. In 4 Japanese placebo-controlled RCTs, risedronate had improved BMD, especially at the lumbar spine, and suppressed BTMs with similar degree of changes in patients with different stages of CKD [89]. Preclinical studies showed that BPs improved bone mass, mineralization, cortical mechanical properties, and bone strength [82]. Toussaint et al. [90] reported that alendronate increased lumbar spine BMD, but not femoral neck $\mathrm{BMD}$, and reduced fracture risk, but not significantly, in women with stage 3-4 CKD. Ward et al. [91] reported BPs improved trabecular bone structure and bone stiffness in 
postmenopausal osteoporotic women up to 16 years. However, data regarding the impact of $\mathrm{BP}$ on bone quality in CKD patients are lacking. Nevertheless, the concern pertaining to development of adynamic bone disease and BPs efficacy and safety in advanced CKD patients remains challenging. KDIGO guideline recommends BP usage in CKD stage 3-5 with biochemical abnormality and low $\mathrm{BMD}$ and/or low trauma fracture with consideration of CKD progression, reversibility of biochemical abnormalities, and bone biopsy if needed [92].

\section{Denosumab}

Denosumab is a monoclonal antibody to receptor activator of nuclear factor $\mathrm{\kappa B}$ ligand (RANKL). It is a potent antiresorptive medication, cleared by the reticuloendothelial system, and does not accumulate in the setting of kidney dysfunction. It should be avoided in patient with CKD and LTBD [93]. Denosumab increases BMD in CKD patients who had received kidney transplantation [94]. Furthermore, it increases BMD and reduces risk of fracture in women with mild to moderate renal failure (CKD stage 2-3). Long-term treatment with denosumab in patients with mild to moderate renal insufficiency did not affect the kidney function and were not associated with higher adverse events, including hypocalcemia, during 7or 10-year treatment [95]. Moreover, in dialysis patients with iPTH $>1,000 \mathrm{pg} / \mathrm{mL}$, treatment with denosumab for 6 months increased both femoral neck and lumbar spine BMDs and significantly decreased iPTH, alkaline phosphatase, calcium-phosphorus product, and bone pain [96]. Kunizawa et al. [97] reported that denosumab was almost equally effective in increasing lumbar spine and femoral neck BMD in both ESRD and CKD patients prior to dialysis. However, the risk of hypocalcemia in CKD patients was higher in advanced stages $[93,95]$, but it was mitigated by its transient and nonserious side effects. To date, there are no studies that have investigated the impact of denosumab on bone morphology in CKD patients. Denosumab in postmenopausal women maintained trabecular and cortical microarchitecture, mineralization, and moreover reduced cortical porosity. Bone histomorphometry showed reduction of bone resorption and decrease in static and dynamic bone formation indices. The reduction in bone turnover was greater than BPs [98].

\section{Gonadal Hormones and Selective Estrogen Receptor}

Modulator

It is evident that CKD patients have a higher rate of fracture and gonadal dysfunction, but there is no consistent correlation between gonadal dysfunction and ROD. In ad- dition, the impact of selective estrogen receptor modulator (SERM) on bone health in CKD patients is not very-well studied. SERM increased BMD and improved bone health in postmenopausal women with CKD [99]. Interestingly, in a recent prospective study, bazedoxifene improved renal function, and phosphate excretion in postmenopausal osteoporotic women without severe renal insufficiency [100]. Moreover, it reduced BTMs, and decreased the fracture rate $[99,101]$. Of note, SERMs and BPs had the same vertebral fracture reduction benefit; however, the beneficial effects of SERMs on BMD and hip fracture were less than BPs [101]. Moreover, transdermal hormone replacement therapy in premenopausal dialysis women has improved lumbar spine BMD over a period of 12 months. On the other hand, testosterone had relatively improved sexual function, without significant beneficial effects on BMD over a short period (6 months) in men on dialysis [99].

\section{Calcitonin and Strontium}

Calcitonin increases renal calcium reabsorption and decreases renal phosphorus reabsorption, which leads to reduction of PTH levels and osteoclastic activity. Calcitonin in rats with moderate CKD reduced osteomalacia [102]. It prevents osteoclast maturation but promotes osteoblast differentiation. It can prevent bone loss in dialysis patients [103]. In HD patients, the effect of combination of intranasal calcitonin and 1-alpha- $(\mathrm{OH})-\mathrm{D} 3$ on increasing BMD and reducing bone resorption marker (serum hydroxyproline) was better than each one alone [104]. Calcitonin prevented bone loss, especially at the lumbar spine, in kidney transplant recipient [105], but had no significant effect on reducing fracture risk [105]. In addition, the beneficial effects of calcitonin on the skeleton gradually disappears because of antibody formation and receptor downregulation [102]. Moreover, the longterm use of calcitonin may increase the risk of cancers [106]. Clinicians must take into the account the little benefits on bone health and the safety profile before prescribing calcitonin. The role of strontium seems to be complex and dose dependent, as low doses of strontium stimulate bone formation, but high doses may cause mineralization defect/osteomalacia in CKD patients [107].

\section{Bone Builders/Anabolics}

Teriparatide and Abaloparatide

Currently, LTBD is the most common ROD pattern in dialysis patients [108]. Teriparatide can potentially have beneficial effects in CKD patients with LTBD. Teriparatide improved lumbar spine BMD, but its impact on femoral neck BMD was inconsistent in both $\mathrm{HD}$ and earlier 
stages of CKD patients $[87,109,110]$. Teriparatide improved bone formation, confirmed by bone biopsy, in an HD patient with LTBD [111]. Daily teriparatide injections are usually well tolerated [112]. However, there is a possible risk of hypotension with weekly injection (higher dose at a time) due to the vasodilatory effect of PTH [109, 110]. Interestingly, 3 times a week $(20 \mu \mathrm{g})$ [113] and weekly $(56.5-\mu \mathrm{g})[109,110]$ teriparatide injections in dialysis, patients showed almost similar benefits. It also improved BTMs and would improve compliance and the safety profile $[109,110]$. Because teriparatide use was associated with dose-dependent increased risk of osteosarcoma in rats, but in humans less than the background incidence rate, its use in humans is usually limited to 18-24 months.

Abaloparatide reduced the incidence of fractures with an acceptable safety profile in a large phase 3 , doubleblinded, placebo-controlled RCT in postmenopausal women with osteoporosis [114]. Similar to teriparatide, abaloparatide reduced fracture (especially vertebral) rates in mild to moderate CKD patients in a post hoc analysis. Interestingly, abaloparatide had a better impact on lumbar spine and femoral neck BMD in patients with eGFR $<60 \mathrm{~mL} / \mathrm{min}$ [115]. In terms of histomorphometry, abaloparatide and teriparatide groups had a lower eroded surface than placebo but only the abaloparatide group reached significance. Cortical porosity was higher in both the abaloparatide and the teriparatide groups than in the placebo group. In addition, there was less pronounced increase in CTX in the abaloparatide group than the teriparatide group [116].

\section{Romosozumab}

CKD progression leads to higher sclerostin levels, which is associated with higher mortality and cardiovascular events [117]. The major source of sclerostin is osteocytes but is secreted by the kidney, liver, and vascular wall too [84]. Romosozumab is a sclerostin monoclonal antibody that increases the bone formation and decreases fracture risk. It would be an interesting approach for CKD patients with LTBD [118]. Antisclerostin antibody in CKD rats improved the trabecular bone volume and mineralization, but without significant improvement of biomechanical properties [119]. There is a concern about increased extraskeletal calcification with its usage, so in CKD patients it should be used with caution, as cardiovascular calcification is a main contributor to cardiovascular morbidity and mortality in CKD patients [118]. Nevertheless, alendronate might have a protective impact on reducing the potential romosozumab cardiovascular adverse events [84].

Improving Bone Quality in CKD Patients

\section{New Therapies}

Anti-FGF23 Antibodies

In early CKD stages, FGF23 oversecretion is a compensatory mechanism to maintain mineral homeostasis. However, FGF23 downregulates vitamin D and is associated with various adverse events in advanced CKD patients $[120,121]$. Burosumab is a monoclonal antibody against FGF23 approved by the FDA for treatment of children [122] and adults [123] with X-linked hypophosphatemia. It improved fractures and increased BTMs compared to placebo [123]. There are potential risks and benefits of using anti-FGF23 antibody therapy in CKD patients. It may lead to hyperphosphatemia in predialysis CKD patients. However, it might pose a lower risk in ESRD patients, as different modalities regulate phosphorus homeostasis. Further studies are needed before using burosumab in CKD patients $[120,121]$.

\section{Cathepsin K Antagonists}

Cathepsin $\mathrm{K}$ antagonists were developed to decrease bone resorption. In an animal study, cathepsin $\mathrm{K}$ inhibitor increased cortical BMD and bone strength [124]. Several clinical trials have demonstrated that cathepsin $\mathrm{K}$ antagonists have a potential role in metabolic bone disorders especially in women with postmenopausal osteoporosis $[125,126]$. Odanacatib increased BMD and suppressed bone resorption, without affecting bone formation markers, in postmenopausal women with low BMD. However, these effects were reversible after its discontinuation [127]. These agents were never studied in CKD patients and did not reach phase IV trials due to a concern of increased cerebrovascular events [128].

It is worth mentioning that, despite none of these new agents were developed specifically for the CKD population, their development highlights an exciting future for the improvement of bone quality in CKD patients. They culminate a departure from treating ROD as a metabolic and hormonal derangement to a cellular and biological bone-centric approach focused on bone cell signaling and interactions. How these potential therapeutic developments are going to affect the skeletal and extraskeletal manifestations of CKD needs to be investigated.

\section{Conclusion}

The bone quality and quantity abnormalities in CKD patients are complex. After confirming the diagnosis of ROD and determining the fracture risk, patients should 
be counseled on lifestyle modifications that are beneficial to their bone. Ultimately, CKD patients should be managed with strategies not only focusing on bone quantity but also bone quality abnormalities. Knowing the pathophysiology of bone damage in the setting of CKD can help in distinguishing patients who might benefit from anabolic versus antiresorptive therapies. The therapeutic developments directed toward improving bone quality in CKD patients are predicted to change nephrology practice patterns and lead to improved quality of life and decreased mortality in our CKD patients.

\section{Acknowledgements}

We thank the Kentucky Nephrology Research Trust for supporting our bone research. Also, acknowledge the International Society of Nephrology for funding Mohamed Abdalbary for his research fellowship.

\section{Statement of Ethics}

The authors have no ethical conflicts to disclose.

\section{Conflict of Interest Statement}

The authors have no conflict of interest to disclose.

\section{Funding Sources}

The authors received no specific funding for this article. No funding for the article design, preparation of the manuscript, or any other article-related work.

\section{Author Contributions}

All authors contributed equally in reviewing the literature and writing the manuscript.

\section{References}

1 Moe S, Drüeke T, Cunningham J, Goodman W, Martin K, Olgaard K, et al. Definition, evaluation, and classification of renal osteodystrophy: a position statement from Kidney Disease: Improving Global Outcomes (KDIGO). Kidney Int. 2006;69(11):1945-53.

2 Wagner J, Jhaveri KD, Rosen L, Sunday S, Mathew AT, Fishbane S. Increased bone fractures among elderly United States hemodialysis patients. Nephrol Dial Transplant. 2014; 29(1):146-51.

3 Wakasugi M, Kazama JJ, Taniguchi M, Wada A, Iseki K, Tsubakihara Y, et al. Increased risk of hip fracture among Japanese hemodialysis patients. J Bone Miner Metab. 2013;31(3):315-21.

4 Moe SM. Renal osteodystrophy or kidney-induced osteoporosis? Curr Osteoporos Rep. 2017;15(3):194-7.

5 Ebeling PR, Daly RM, Kerr DA, Kimlin MG. Building healthy bones throughout life: an evidence-informed strategy to prevent osteoporosis in Australia. Med J Aust. 2013;199(7 Suppl):S1-46.

6 Roshanravan B, Gamboa J, Wilund K. Exercise and CKD: skeletal muscle dysfunction and practical application of exercise to prevent and treat physical impairments in CKD. Am J Kidney Dis. 2017;69(6):837-52.

7 Aleksova J, Ng KW, Jung C, Zeimer H, Dwyer $\mathrm{KM}$, Milat F, et al. Bone health in chronic kidney disease-mineral and bone disorder: a clinical case seminar and update. Intern Med J. 2018;48(12):1435-46.

8 Barbosa AP, Lourenço JD, Junqueira JJM, Larissa Emidio de França S, Martins JS, Oliveira Junior MC, et al. The deleterious effects of smoking in bone mineralization and fibrillar matrix composition. Life Sci. 2020;241: 117132.
9 Santos GDD, Elias RM, Dalboni MA, Silva GVD, Moysés RMA. Chronic kidney disease patients who smoke have higher serum phosphorus. J Bras Nefrol. 2019 Apr-Jun;41(2): 288-92.

10 Asadipooya K, Graves L, Greene LW. Transient osteoporosis of the hip: review of the literature. Osteoporos Int. 2017;28(6):180516.

11 Bennett PN, Capdarest-Arest N, Parker K. The physical deterioration of dialysis patients-Ignored, ill-reported, and ill-treated. Semin Dial. 2017;30(5):409-12.

12 Gibbons RJ, Balady GJ, Bricker JT, Chaitman BR, Fletcher GF, Froelicher VF, et al. ACC/ AHA 2002 guideline update for exercise testing: summary article: a report of the American College of Cardiology/American Heart Association Task Force on Practice Guidelines (Committee to Update the 1997 Exercise Testing Guidelines). Circulation. 2002;106(14):1883-92.

13 Liao MT, Liu WC, Lin FH, Huang CF, Chen SY, Liu CC, et al. Intradialytic aerobic cycling exercise alleviates inflammation and improves endothelial progenitor cell count and bone density in hemodialysis patients. Medicine. 2016;95(27):e4134.

14 Grzegorzewska AE, Młot-Michalska M. Bone mineral density, its predictors, and outcomes in peritoneal dialysis patients. Adv Perit Dial. 2011;27:140-5.

15 Grzegorzewska AE, Młot-Michalska M. Total body mass is better than body mass index as a prognostic parameter for bone mineral density in dialyzed patients. Adv Perit Dial. 2009; 25:178-80.

16 Fournie C, Pelletier S, Bacchetta J, Boutroy S, Confavreux C, Drai J, et al. The relationship between body composition and bone quality measured with HR-pQCT in peritoneal dialysis patients. Perit Dial Int. 2017;37(5):548-55.

17 Johansen KL, Painter PL, Sakkas GK, Gordon P, Doyle J, Shubert T. Effects of resistance exercise training and nandrolone decanoate on body composition and muscle function among patients who receive hemodialysis: a randomized, controlled trial. J Am Soc Nephrol. 2006;17(8):2307-14.

18 Marinho SM, Mafra D, Pelletier S, Hage V, Teuma C, Laville M, et al. Hemodialysis patients, intradialytic resistance exercise improves osteoblast function: a pilot study. J Ren Nutr. 2016;26(5):341-5.

19 Marinho SM, Moraes C, Barbosa JE, Carraro Eduardo JC, Fouque D, Pelletier S, et al. Exercise training alters the bone mineral density of hemodialysis patients. J Strength Cond Res. 2016;30(10):2918-23.

20 Maimoun L, Sultan C. Effect of physical activity on calcium homeostasis and calciotropic hormones: a review. Calcif Tissue Int. 2009; 85(4):277-86.

21 Scott A, Khan KM, Duronio V, Hart DA. Mechanotransduction in human bone: in vitro cellular physiology that underpins bone changes with exercise. Sports Med. 2008;38(2):139-60.

22 Zaluska A, Zaluska WT, Bednarek-Skublewska A, Ksiazek A. Nutrition and hydration status improve with exercise training using stationary cycling during hemodialysis (HD) in patients with end-stage renal disease (ESRD). Ann Univ Mariae Curie Sklodowska Med. 2002;57(2):342-6.

23 Parsons TL, Toffelmire EB, King-VanVlack $\mathrm{CE}$. The effect of an exercise program during hemodialysis on dialysis efficacy, blood pressure and quality of life in end-stage renal disease (ESRD) patients. Clin Nephrol. 2004; 61(4):261-74. 
24 Orcy R, Antunes MF, Schiller T, Seus T, Böhlke M. Aerobic exercise increases phosphate removal during hemodialysis: a controlled trial. Hemodial Int. 2014;18(2):450-8.

25 Malluche $\mathrm{HH}$, Monier-Faugere MC, Blomquist G, Davenport DL. Two-year cortical and trabecular bone loss in CKD-5D: biochemical and clinical predictors. Osteoporos Int. 2018;29(1):125-34.

26 Gomes TS, Aoike DT, Baria F, Graciolli FG, Moyses RMA, Cuppari L. Effect of aerobic exercise on markers of bone metabolism of overweight and obese patients with chronic kidney disease. J Ren Nutr. 2017;27(5):364-71.

27 Liao HW, Huang TH, Chang YH, Liou HH, Chou YH, Sue YM, et al. Exercise alleviates osteoporosis in rats with mild chronic kidney disease by decreasing sclerostin production. Int J Mol Sci. 2019;20(8):2044

28 Rodriguez M, Aguilera-Tejero E. Energydense diets and mineral metabolism in the context of chronic kidney disease(-)metabolic bone disease (CKD-MBD). Nutrients. 2018 10(12): 1840 .

29 Kawamoto K, Sakuma M, Tanaka S, Masuda M, Nakao-Muraoka M, Niida Y, et al. Highfat diets provoke phosphorus absorption from the small intestine in rats. Nutrition. 2020;72:110694.

30 Phillips CM, Chen LW, Heude B, Bernard JY, Harvey NC, Duijts L, et al. Dietary inflammatory index and non-communicable disease risk: a narrative review. Nutrients. 2019 Aug 12;11(8):1873.

31 Bach KE, Kelly JT, Palmer SC, Khalesi S, Strippoli GFM, Campbell KL. Healthy dietary patterns and incidence of CKD: a meta-analysis of cohort studies. Clin J Am Soc Nephrol. 2019;14(10):1441-9.

32 Rahbar A, Larijani B, Nabipour I, Mohamadi MM, Mirzaee K, Amiri Z. Relationship among dietary estimates of net endogenous acid production, bone mineral density and biochemical markers of bone turnover in an Iranian general population. Bone. 2009;45(5):876-81.

33 Jia T, Byberg L, Lindholm B, Larsson TE, Lind L, Michaëlsson K, et al. Dietary acid load, kidney function, osteoporosis, and risk of fractures in elderly men and women. Osteoporos Int. 2015;26(2):563-70.

34 Shams-White MM, Chung M, Fu Z, Insogna KL, Karlsen MC, LeBoff MS, et al. Animal versus plant protein and adult bone health: a systematic review and meta-analysis from the National Osteoporosis Foundation. PLoS One. 2018;13(2):e0192459.

35 Ke G, Huang J, Zhu Y, Yang J, Zhang Y, Chen $\mathrm{L}$, et al. Effect of ascorbic acid on mineral and bone disorders in hemodialysis patients: a systematic review and meta-analysis. Kidney Blood Press Res. 2018;43(5):1459-71.

36 Chewcharat A, Takkavatakarn K, Wongrattanagorn S, Panrong K, Kittiskulnam P, Eiam-Ong S, et al. The effects of restricted protein diet supplemented with ketoanalogue on renal function, blood pressure, nutritional status, and chronic kidney disease-mineral and bone disorder in chronic kidney disease patients: a systematic review and meta-analysis. J Ren Nutr. 2019 May;30(3):189-99.

37 Koppe L, Cassani de Oliveira M, Fouque D. Ketoacid analogues supplementation in chronic kidney disease and future perspectives. Nutrients. 2019 Sep 3;11(9):2071.

38 Chauveau P, Koppe L, Combe C, Lasseur C, Trolonge S, Aparicio M. Vegetarian diets and chronic kidney disease. Nephrol Dial Transplant. 2019;34(2):199-207.

39 Aloia J, Bojadzievski T, Yusupov E, Shahzad G, Pollack S, Mikhail M, et al. The relative influence of calcium intake and vitamin D status on serum parathyroid hormone and bone turnover biomarkers in a double-blind, placebo-controlled parallel group, longitudinal factorial design. J Clin Endocrinol Metab. 2010;95(7):3216-24.

40 Zhao JG, Zeng XT, Wang J, Liu L. Association between calcium or vitamin D supplementation and fracture incidence in communitydwelling older adults: a systematic review and meta-analysis. JAMA. 2017;318(24):2466-82

41 Spiegel DM, Brady K. Calcium balance in normal individuals and in patients with chronic kidney disease on low- and high-calcium diets. Kidney Int. 2012;81(11):1116-22.

42 Hill KM, Martin BR, Wastney ME, McCabe GP, Moe SM, Weaver CM, et al. Oral calcium carbonate affects calcium but not phosphorus balance in stage 3-4 chronic kidney disease. Kidney Int. 2013;83(5):959-66.

43 Moe SM. Calcium as a cardiovascular toxin in CKD-MBD. Bone. 2017;100:94-9.

44 Fusaro M, Holden R, Lok C, Iervasi G, Plebani M, Aghi A, et al. Phosphate and bone fracture risk in chronic kidney disease patients. Nephrol Dial Transplant. 2021 Feb 20; 36(3):405-12.

45 Campos-Obando N, Koek WNH, Hooker ER, van der Eerden BC, Pols HA, Hofman A, et al. Serum phosphate is associated with fracture risk: the Rotterdam study and MrOS. J Bone Miner Res. 2017;32(6):1182-93.

46 Rojas E, Carlini RG, Clesca P, Arminio A, Suniaga $\mathrm{O}$, De Elguezabal $\mathrm{K}$, et al. The pathogenesis of osteodystrophy after renal transplantation as detected by early alterations in bone remodeling. Kidney Int. 2003;63(5):1915-23.

47 Raggi P, James G, Burke SK, Bommer J, Chasan-Taber S, Holzer H, et al. Decrease in thoracic vertebral bone attenuation with calcium-based phosphate binders in hemodialysis. J Bone Miner Res. 2005;20(5):764-72.

48 Block GA, Wheeler DC, Persky MS, Kestenbaum B, Ketteler M, Spiegel DM, et al. Effects of phosphate binders in moderate CKD. J Am Soc Nephrol. 2012;23(8):1407-15.

49 Malluche HH, Siami GA, Swanepoel C, Wang $\mathrm{GH}$, Mawad H, Confer S, et al. Improvements in renal osteodystrophy in patients treated with lanthanum carbonate for two years. Clin Nephrol. 2008;70(4):284-95.

50 Barreto DV, Barreto FC, de Carvalho AB, Cuppari L, Draibe SA, Dalboni MA, et al. Phosphate binder impact on bone remodeling and coronary calcification: results from the BRiC study. Nephron Clin Pract. 2008;110(4): c273-83.

51 Ferreira A, Frazão JM, Monier-Faugere MC, Gil C, Galvao J, Oliveira C, et al. Effects of sevelamer hydrochloride and calcium carbonate on renal osteodystrophy in hemodialysis patients. J Am Soc Nephrol. 2008;19(2): 405-12.

52 Shigematsu T, Tokumoto A, Nakaoka A, Arisaka $\mathrm{H}$. Effect of lanthanum carbonate treatment on bone in Japanese dialysis patients with hyperphosphatemia. Ther Apher Dial. 2011;15(2):176-84

53 Yajima A, Inaba $M$, Tominaga $Y$, Tanaka $M$, Otsubo S, Nitta K, et al. Impact of lanthanum carbonate on cortical bone in dialysis patients with adynamic bone disease. Ther Apher Dial. 2013;17(Suppl 1):41-8.

54 Goto K, Goto S, Fujii H, Watanabe K, Kono $\mathrm{K}$, Nishi S. Effects of lanthanum carbonate on bone markers and bone mineral density in incident hemodialysis patients. J Bone Miner Metab. 2019;37(6):1075-82.

55 Zhang C, Wang S, Zhao S, Zhang X. Effect of lanthanum carbonate on coronary artery calcification and bone mineral density in maintenance hemodialysis patients with diabetes complicated with adynamic bone disease: a prospective pilot study. Medicine. 2017; 96(45):e8664.

56 Zhang C, Wen J, Li Z, Fan J. Efficacy and safety of lanthanum carbonate on chronic kidney disease-mineral and bone disorder in dialysis patients: a systematic review. BMC Nephrol. 2013;14:226.

57 Bouillon R, Marcocci C, Carmeliet G, Bikle D, White JH, Dawson-Hughes B, et al. Skeletal and extraskeletal actions of vitamin D: current evidence and outstanding questions. Endocr Rev. 2019;40(4):1109-51.

58 Jean G, Souberbielle JC, Chazot C. Vitamin D in chronic kidney disease and dialysis patients. Nutrients. 2017;9(4):328.

59 Nigwekar SU, Tamez H, Thadhani RI. Vitamin $\mathrm{D}$ and chronic kidney disease-mineral bone disease (CKD-MBD). Bonekey Rep. 2014;3:498.

60 Jean G, Terrat JC, Vanel T, Hurot JM, Lorriaux C, Mayor B, et al. Evidence for persistent vitamin D 1-alpha-hydroxylation in hemodialysis patients: evolution of serum 1,25-dihydroxycholecalciferol after 6 months of 25-hydroxycholecalciferol treatment. Nephron Clin Pract. 2008;110(1): c58-65.

61 Kalantar-Zadeh K, Miller JE, Kovesdy CP, Mehrotra R, Lukowsky LR, Streja E, et al. Impact of race on hyperparathyroidism, mineral disarrays, administered vitamin $\mathrm{D}$ mimetic, and survival in hemodialysis patients. J Bone Miner Res. 2010;25(12):2724-34.

62 Stehman-Breen CO, Sherrard D, Walker A, Sadler R, Alem A, Lindberg J. Racial differences in bone mineral density and bone loss among end-stage renal disease patients. Am J Kidney Dis. 1999;33(5):941-6. 
63 Mathew AT, Hazzan A, Jhaveri KD, Block GA, Chidella S, Rosen L, et al. Increasing hip fractures in patients receiving hemodialysis and peritoneal dialysis. Am J Nephrol. 2014; 40(5):451-7.

64 Toussaint ND, Damasiewicz MJ. Do the benefits of using calcitriol and other vitamin D receptor activators in patients with chronic kidney disease outweigh the harms? Nephrology. 2017;22(Suppl 2):51-6.

65 Trillini M, Cortinovis M, Ruggenenti P, Reyes Loaeza J, Courville K, Ferrer-Siles C, et al. Paricalcitol for secondary hyperparathyroidism in renal transplantation. J Am Soc Nephrol. 2015;26(5):1205-14.

66 Wijenayaka AR, Yang D, Prideaux M, Ito N, Kogawa M, Anderson $\mathrm{PH}$, et al. 1a,25dihydroxyvitamin D3 stimulates human SOST gene expression and sclerostin secretion. Mol Cell Endocrinol. 2015;413:157-67.

67 Baker LR, Abrams L, Roe CJ, Faugere MC, Fanti P, Subayti Y, et al. 1,25(OH)2D3 administration in moderate renal failure: a prospective double-blind trial. Kidney Int. 1989; 35(2):661-9.

68 Cai P, Tang X, Qin W, Ji L, Li Z. Comparison between paricalcitol and active non-selective vitamin D receptor activator for secondary hyperparathyroidism in chronic kidney disease: a systematic review and meta-analysis of randomized controlled trials. Int Urol Nephrol. 2016;48(4):571-84.

69 Vondracek SF, Hoody DW. Combination vitamin D therapy in stage 5 chronic kidney disease. Ann Pharmacother. 2011;45(7-8): 1011-5.

70 Cunningham J, Locatelli F, Rodriguez M. Secondary hyperparathyroidism: pathogenesis, disease progression, and therapeutic options. Clin J Am Soc Nephrol. 2011;6(4):913-21.

71 Malluche HH, Monier-Faugere MC, Wang G, Frazã O JM, Charytan C, Coburn JW, et al. An assessment of cinacalcet $\mathrm{HCl}$ effects on bone histology in dialysis patients with secondary hyperparathyroidism. Clin Nephrol. 2008; 69(4):269-78.

72 Sekercioglu N, Busse JW, Sekercioglu MF, Agarwal A, Shaikh S, Lopes LC, et al. Cinacalcet versus standard treatment for chronic kidney disease: a systematic review and metaanalysis. Ren Fail. 2016;38(6):857-74.

73 Wetmore JB, Gurevich K, Sprague S, Da Roza G, Buerkert J, Reiner M, et al. A randomized trial of cinacalcet versus vitamin $\mathrm{D}$ analogs as monotherapy in secondary hyperparathyroidism (PARADIGM). Clin J Am Soc Nephrol. 2015;10(6):1031-40.

74 Palmer SC, Mavridis D, Johnson DW, Tonelli M, Ruospo M, Strippoli GFM. Comparative effectiveness of calcimimetic agents for secondary hyperparathyroidism in adults: a systematic review and network meta-analysis. Am J Kidney Dis. 2020;76(3):321-30.

75 Block GA, Bushinsky DA, Cunningham J, Drueke TB, Ketteler M, Kewalramani R, et al. Effect of etelcalcetide vs placebo on serum parathyroid hormone in patients receiving hemodialysis with secondary hyperparathyroidism: two randomized clinical trials. JAMA. 2017;317(2):146-55.

76 Block GA, Bushinsky DA, Cheng S, Cunningham J, Dehmel B, Drueke TB, et al. Effect of etelcalcetide vs cinacalcet on serum parathyroid hormone in patients receiving hemodialysis with secondary hyperparathyroidism: a randomized clinical trial. JAMA. 2017;317(2): 156-64.

77 Bushinsky DA, Chertow GM, Cheng S, Deng H, Kopyt N, Martin KJ, et al. One-year safety and efficacy of intravenous etelcalcetide in patients on hemodialysis with secondary hyperparathyroidism. Nephrol Dial Transplant. 2020 Oct 1;35(10):1769-78.

78 Russo D, Tripepi R, Malberti F, Di Iorio B, Scognamiglio B, Di Lullo L, et al. Etelcalcetide in patients on hemodialysis with severe secondary hyperparathyroidism. Multicenter study in "real life". J Clin Med. 2019;8(7): 1066.

79 Shigematsu T, Fukagawa M, Yokoyama K, Akiba T, Fujii A, Odani M, et al. Effects of the intravenous calcimimetic etelcalcetide on bone turnover and serum fibroblast growth factor 23: post hoc analysis of an open-label study. Clin Ther. 2018;40(12): 2099-111.

80 Diaz-Tocados JM, Rodriguez-Ortiz ME, Almaden Y, Pineda C, Martinez-Moreno JM, Herencia $\mathrm{C}$, et al. Calcimimetics maintain bone turnover in uremic rats despite the concomitant decrease in parathyroid hormone concentration. Kidney Int. 2019;95(5):106478.

81 Chen J, Budoff MJ, Reilly MP, Yang W, Rosas SE, Rahman M, et al. Coronary artery calcification and risk of cardiovascular disease and death among patients with chronic kidney disease. JAMA Cardiol. 2017;2(6): 635-43.

82 Damasiewicz MJ, Nickolas TL. Bisphosphonate therapy in CKD: the current state of affairs. Curr Opin Nephrol Hypertens. 2020; 29(2):221-6.

83 Yamasaki M, Yuasa T, Uehara S, Fujii Y, Yamamoto $\mathrm{S}$, Masuda $\mathrm{H}$, et al. Improvement of renal function by changing the bone-modifying agent from zoledronic acid to denosumab. Int J Clin Oncol. 2016;21(6):1191-5.

84 Asadipooya K, Weinstock A. Cardiovascular outcomes of romosozumab and protective role of alendronate. Arterioscler Thromb Vasc Biol. 2019;39(7):1343-50.

85 Alarkawi D, Ali MS, Bliuc D, Pallares N, Tebe C, Elhussein L, et al. Oral bisphosphonate use and all-cause mortality in patients with moderate-severe (grade 3B-5D) chronic kidney disease: a population-based cohort study. J Bone Miner Res. 2020 May;35(5): 894-900.

86 Toth-Manikowski SM, Francis JM, Gautam A, Gordon CE. Outcomes of bisphosphonate therapy in kidney transplant recipients: a systematic review and meta-analysis. Clin Transplant. 2016;30(9):1090-6.
87 Wilson LM, Rebholz CM, Jirru E, Liu MC, Zhang A, Gayleard J, et al. Benefits and harms of osteoporosis medications in patients with chronic kidney disease: a systematic review and meta-analysis. Ann Intern Med. 2017; 166(9):649-58.

88 Miller PD, Roux C, Boonen S, Barton IP, Dunlap LE, Burgio DE. Safety and efficacy of risedronate in patients with age-related reduced renal function as estimated by the Cockcroft and Gault method: a pooled analysis of nine clinical trials. J Bone Miner Res. 2005;20(12):2105-15.

89 Sugimoto T, Inoue D, Maehara M, Oikawa I, Shigematsu T, Nishizawa Y. Efficacy and safety of once-monthly risedronate in osteoporosis subjects with mild-to-moderate chronic kidney disease: a post hoc subgroup analysis of a phase III trial in Japan. J Bone Miner Metab. 2019;37(4):730-40.

90 Toussaint ND, Lau KK, Strauss BJ, Polkinghorne KR, Kerr PG. Effect of alendronate on vascular calcification in CKD stages 3 and 4: a pilot randomized controlled trial. Am J Kidney Dis. 2010;56(1):57-68.

91 Ward J, Wood C, Rouch K, Pienkowski D, Malluche HH. Stiffness and strength of bone in osteoporotic patients treated with varying durations of oral bisphosphonates. Osteoporos Int. 2016;27(9):2681-8.

92 Ketteler M, Block GA, Evenepoel P, Fukagawa M, Herzog CA, McCann L, et al. Executive summary of the 2017 KDIGO Chronic Kidney Disease-Mineral and Bone Disorder (CKDMBD) Guideline Update: what's changed and why it matters. Kidney Int. 2017;92(1):26-36.

93 Miller PD. Chronic kidney disease and osteoporosis: evaluation and management. Bonekey Rep. 2014;3:542.

94 Thongprayoon C, Acharya P, Aeddula NR, Torres-Ortiz A, Bathini T, Sharma K, et al. Effects of denosumab on bone metabolism and bone mineral density in kidney transplant patients: a systematic review and meta-analysis. Arch Osteoporos. 2019;14(1):35.

95 Broadwell A, Chines A, Ebeling PR, Franek E, Huang S, Smith S, et al. Denosumab safety and efficacy among participants in the FREEDOM extension study with mild to moderate chronic kidney disease. J Clin Endocrinol Metab. 2021;106(2):397-409.

96 Chen CL, Chen NC, Hsu CY, Chou KJ, Lee PT, Fang HC, et al. An open-label, prospective pilot clinical study of denosumab for severe hyperparathyroidism in patients with low bone mass undergoing dialysis. J Clin Endocrinol Metab. 2014;99(7):2426-32.

97 Kunizawa K, Hiramatsu R, Hoshino J, Mizuno H, Ozawa Y, Sekine A, et al. Denosumab for dialysis patients with osteoporosis: a cohort study. Sci Rep. 2020;10(1):2496.

98 Brown JP, Reid IR, Wagman RB, Kendler D, Miller PD, Jensen JE, et al. Effects of up to 5 years of denosumab treatment on bone histology and histomorphometry: the FREEDOM study extension. J Bone Miner Res. 2014; 29(9):2051-6. 
99 Aleksova J, Rodriguez AJ, McLachlan R, Kerr P, Milat F, Ebeling PR. Gonadal hormones in the pathogenesis and treatment of bone health in patients with chronic kidney disease: a systematic review and meta-analysis. Curr Osteoporos Rep. 2018;16(6):67492.

100 Masaki H, Imanishi Y, Naka H, Nagata Y, Kurajoh M, Mori K, et al. Bazedoxifene improves renal function and increases renal phosphate excretion in patients with postmenopausal osteoporosis. J Bone Miner Metab. 2020 May;38(3):405-11.

101 Adami S, Palacios S, Rizzoli R, Levine AB, Sutradhar S, Chines AA. The efficacy and safety of bazedoxifene in postmenopausal women by baseline kidney function status. Climacteric. 2014;17(3):273-84.

102 Gordon PL, Frassetto LA. Management of osteoporosis in CKD Stages 3 to 5. Am J Kidney Dis. 2010;55(5):941-56.

103 Wang SX, Li H. Salmon calcitonin in prevention of osteoporosis in maintenance dialysis patients. Chin Med J. 2008;121(14): 1280-4.

104 Matuszkiewicz-Rowinska J, Niemczyk S, Puka J, Sawicki A, Switalski M, Talalaj M, et al. [Combined therapy with calcitonin and high doses of active vitamin D3 metabolites in uremic hyperparathyroidism]. Pol Arch Med Wewn. 1996;96(1):23-31.

105 Palmer SC, Strippoli GF, McGregor DO. Interventions for preventing bone disease in kidney transplant recipients: a systematic review of randomized controlled trials. Am J Kidney Dis. 2005;45(4):638-49.

106 Wells G, Chernoff J, Gilligan JP, Krause DS. Does salmon calcitonin cause cancer? A review and meta-analysis. Osteoporos Int. 2016;27(1):13-9.

107 Schrooten I, Behets GJ, Cabrera WE, Vercauteren SR, Lamberts LV, Verberckmoes SC, et al. Dose-dependent effects of strontium on bone of chronic renal failure rats. Kidney Int. 2003;63(3):927-35.

108 Malluche HH, Mawad HW, Monier-Faugere MC. Renal osteodystrophy in the first decade of the new millennium: analysis of 630 bone biopsies in black and white patients. J Bone Miner Res. 2011;26(6):1368-76.

109 Yamamoto J, Nakazawa D, Nishio S, Ishikawa Y, Makita M, Kusunoki Y, et al. Impact of weekly teriparatide on the bone and mineral metabolism in hemodialysis patients with relatively low serum parathyroid hormone: a pilot study. Ther Apher Dial. 2020;24(2):146-53.
110 Sumida K, Ubara Y, Hoshino J, Mise K, Hayami N, Suwabe T, et al. Once-weekly teriparatide in hemodialysis patients with hypoparathyroidism and low bone mass: a prospective study. Osteoporos Int. 2016;27(4): 1441-50.

111 Palcu P, Dion N, Ste-Marie LG, Goltzman D, Radziunas I, Miller PD, et al. Teriparatide and bone turnover and formation in a hemodialysis patient with low-turnover bone disease: a case report. Am J Kidney Dis. 2015;65(6):933-6.

112 Nishikawa A, Yoshiki F, Taketsuna M, Kajimoto K, Enomoto H. Safety and effectiveness of daily teriparatide for osteoporosis in patients with severe stages of chronic kidney disease: post hoc analysis of a postmarketing observational study. Clin Interv Aging. 2016;11:1653-9.

113 Mitsopoulos E, Ginikopoulou E, Economidou D, Zanos S, Pateinakis P, Minasidis E, et al. Impact of long-term cinacalcet, ibandronate or teriparatide therapy on bone mineral density of hemodialysis patients: a pilot study. Am J Nephrol. 2012;36(3):238-44.

114 Miller PD, Hattersley G, Riis BJ, Williams GC, Lau E, Russo LA, et al. Effect of abaloparatide vs placebo on new vertebral fractures in postmenopausal women with osteoporosis: a randomized clinical trial. JAMA. 2016;316(7):722-33.

115 Bilezikian JP, Hattersley G, Mitlak BH, Hu MY, Fitzpatrick LA, Dabrowski C, et al. Abaloparatide in patients with mild or moderate renal impairment: results from the ACTIVE phase 3 trial. Curr Med Res Opin. 2019;35(12):2097-102.

116 Moreira CA, Fitzpatrick LA, Wang Y, Recker RR. Effects of abaloparatide-SC (BA058) on bone histology and histomorphometry: the ACTIVE phase 3 trial. Bone. 2017;97: 314-9.

117 Kanbay M, Siriopol D, Saglam M, Kurt YG, Gok M, Cetinkaya H, et al. Serum sclerostin and adverse outcomes in nondialyzed chronic kidney disease patients. J Clin Endocrinol Metab. 2014;99(10):E1854-61.

118 Brandenburg VM, Verhulst A, Babler A, D'Haese PC, Evenepoel P, Kaesler N. Sclerostin in chronic kidney disease-mineral bone disorder think first before you block it! Nephrol Dial Transplant. 2019;34(3):40814.
119 Moe SM, Chen NX, Newman CL, Organ JM, Kneissel M, Kramer I, et al. Anti-sclerostin antibody treatment in a rat model of progressive renal osteodystrophy. J Bone Miner Res. 2015;30(3):499-509.

120 Perwad F, Portale AA. Burosumab therapy for X-linked hypophosphatemia and therapeutic implications for CKD. Clin J Am Soc Nephrol. 2019;14(7):1097-9.

121 Fukumoto S. Targeting fibroblast growth factor 23 signaling with antibodies and inhibitors, is there a rationale? Front Endocrinol. 2018;9:48.

122 Carpenter TO, Whyte MP, Imel EA, Boot AM, Högler W, Linglart A, et al. Burosumab therapy in children with X-linked hypophosphatemia. N Engl J Med. 2018;378(21): 1987-98.

123 Insogna KL, Briot K, Imel EA, Kamenický P, Ruppe MD, Portale AA, et al. A Randomized, double-blind, placebo-controlled, phase 3 trial evaluating the efficacy of burosumab, an anti-FGF23 antibody, in adults with X-linked hypophosphatemia: week 24 primary analysis. J Bone Miner Res. 2018; 33(8):1383-93.

124 Yamada H, Ochi Y, Mori H, Nishikawa S, Hashimoto Y, Tanaka M, et al. Cortical bone mineral density is increased by the cathepsin $\mathrm{K}$ inhibitor ONO-5334, which leads to a robust increase in bone strength: results from a 16-month study in ovariectomised cynomolgus monkeys. J Bone Miner Metab. 2019;37(4):636-47.

125 Stone JA, McCrea JB, Witter R, Zajic S, Stoch SA. Clinical and translational pharmacology of the cathepsin $\mathrm{K}$ inhibitor odanacatib studied for osteoporosis. Br J Clin Pharmacol. 2019;85(6):1072-83.

126 Tanaka M, Hashimoto Y, Hasegawa C, Deacon S, Eastell R. Antiresorptive effect of a cathepsin $\mathrm{K}$ inhibitor ONO-5334 and its relationship to BMD increase in a phase II trial for postmenopausal osteoporosis. BMC Musculoskelet Disord. 2017;18(1):267.

127 Eisman JA, Bone HG, Hosking DJ, McClung MR, Reid IR, Rizzoli R, et al. Odanacatib in the treatment of postmenopausal women with low bone mineral density: three-year continued therapy and resolution of effect. J Bone Miner Res. 2011;26(2):242-51.

128 Khairallah P, Nickolas TL. Updates in CKDassociated osteoporosis. Curr Osteoporos Rep. 2018;16(6):712-23. 